\title{
New Exact Solutions and Novel Localized Excitations for the (2+1)-Dimensional Broek-Kaup System with Variable Coefficients
}

\author{
Song-Hua Ma, Jian-Ping Fang, and Bi-Hai Hong \\ Department of Physics, Zhejiang Lishui University, Lishui 323000, China \\ Reprint requests to S.-H. M.; E-mail: msh6209@yahoo.com.cn
}

Z. Naturforsch. 64a, 795 - 800 (2009); received March 3, 2009

In this paper, a new projective equation $\left(\phi^{\prime}=\sigma \phi+\phi^{2}\right)$ is used to obtain the variable separation solutions with two arbitrary functions of the (2+1)-dimensional Broek-Kaup system with variable coefficients (VCBK). Based on the derived solutions and by selecting appropriate functions, some novel folded solitary wave evolutional behaviours are investigated.

Key words: New Projective Equation $\left(\phi^{\prime}=\sigma \phi+\phi^{2}\right)$; Variable Separation Solutions;

(2+1)-Dimensional VCBK System; Folded Solitary Wave Evolutional Behaviours.

PACS numbers: 05.45.Yv; 03.65.Ge

\section{Introduction}

Over the past 30 years the concept of solitons has entered into various branches of many natural science such as chemistry, biology, mathematics, communication, and in particular in almost all branches of physics like fluid dynamics, plasma physics, field theory, optics, and condensed matter physics [1-7]. Searching for an analytical exact solution to a nonlinear system has long been an important and interesting topic in nonlinear science both for physicists and mathematicians, and various methods for obtaining exact solutions of a nonlinear system have been proposed, for example, the bilinear method [8], the standard Painlevé truncated expansion [9], the method of "coalescence of eigenvalue" or "wavenumbers" [10], the homogenous balance method [11], and the mapping method [12], etc. The mapping approach is a kind of classic, efficient, and well-developed method to solve nonlinear evolution equations, the remarkable characteristic of which is that we can have many different ansatzs and therefore, a large number of solutions.

In the past, we have found the exact solutions of some nonlinear systems via the Ricatti equation $\left(\phi^{\prime}=\sigma+\phi^{2}\right)$ mapping method, such as $(1+1)$ dimensional related Schrödinger equation, $(2+1)$-dimensional Broer-Kaup-Kupershmidt system, (2+1)-dimensional Generalized Broer-Kaup system, (3+1)-dimensional Burgers system, (3+1)-dimensional JimboMiwa system, $(2+1)$-dimensional modified dispersive water-wave system, (2+1)-dimensional Boiti-Leon-
Pempinelli system, (2+1)-dimensional Korteweg-de Vries system, (2+1)-dimensional asymmetric NizhnikNovikov-Veselov system, etc. [13-22]. In this paper, with a new projective equation $\left(\phi^{\prime}=\sigma \phi+\phi^{2}\right)$ and a linear variable separation approach, a new family of exact solutions with arbitrary functions of the $(2+1)$ dimensional Broek-Kaup system with variable coefficients (VCBK) is derived and based on the derived solutions, we find a few novel folded solitary wave excitation.

The (2+1)-dimensional VCBK system is

$$
\begin{aligned}
& u_{t y}-a(t)\left[u_{x x y}-2\left(u u_{x}\right)_{y}-2 v_{x x}\right]=0, \\
& v_{t}+a(t)\left[v_{x x}+2(u v)_{x}\right]=0,
\end{aligned}
$$

where $a(t)$ is an arbitrary function of time $t$. It is evident that when $a(t)=1$, the VCBK system will be degenerated to the well-know $(2+1)$-dimensional Broer-Kaup-Kupershmidt (BKK) system, which may be derived from the inner parameter dependent symmetry constant of the Kadomtsev-petviashvili model. When $y=x$, the $(2+1)$-dimensional Broer-KaupKupershmidt system is reduced to the usual $(1+1)$ dimensional BKK system, which is often used to describe the propagation of long waves in shallow water [23]. Using some suitable dependent and independent variable transformations, the $(2+1)$-dimensional BKK system can be further transformed to the $(2+1)$ dimensional dispersive long-water wave equation and $(2+1)$-dimensional Ablowitz-Naup-Newell-Segur system [24]. Actually the (2+1)-dimensional BKK sys- 
tem has been widely investigated in details by many researcher [25].

The basic idea of the new equation $\left(\phi^{\prime}=\sigma \phi+\phi^{2}\right)$ mapping approach is as follows. For a given nonlinear partial differential equation (NPDE) with the independent variables $x=\left(x_{0}=t, x_{1}, x_{2}, \cdots, x_{m}\right)$, and the dependent variable $u$, in the form

$$
P\left(u, u_{t}, u_{x_{i}}, u_{x_{i} x_{j}}, \cdots\right)=0,
$$

where $P$ is in general a polynomial function of its arguments, and the subscripts denote the partial derivatives, the solution may be assumed to be in the form

$$
u=\sum_{i=0}^{n}\left\{A_{i}(x) \phi^{i}[q(x)]\right\}
$$

with

$$
\phi^{\prime}=\sigma \phi+\phi^{2},
$$

where $A_{i}(x)$ and $q(x)$ are functions of the indicated argument to be determined, $\sigma$ is an arbitrary constant, and the prime denotes $\phi$ differentiation with respect to $q$. To determine $u$ explicitly, one substitutes (3) and (4) into the given NPDE and collects the coefficients of the polynomials of $\phi$, then eliminates each coefficient to derive a set of partial differential equations for $A_{i}$ and $q$, and solves the system of partial differential equations to obtain $A_{i}$ and $q$. Finally, (4) is known to possess the general solutions

$$
\phi= \begin{cases}-\frac{1}{2} \sigma\left[1+\tanh \left(\frac{1}{2} \sigma q\right)\right], & \sigma \neq 0, \\ -\frac{1}{2} \sigma\left[1+\operatorname{coth}\left(\frac{1}{2} \sigma q\right)\right], & \sigma \neq 0 \\ -\frac{1}{2} \sigma\left[1-\tan \left(\frac{1}{2} \sigma q\right)\right], & \sigma \neq 0 \\ -\frac{1}{2} \sigma\left[1+\cot \left(\frac{1}{2} \sigma q\right)\right], & \sigma \neq 0 \\ \frac{-1}{q}, & \sigma=0\end{cases}
$$

Substituting $A_{i}, q$, and (5) into (3), one obtains the exact solutions to the given NPDE.

\section{New Exact Solutions of the (2+1)-Dimensional VCBK System}

First, let us make a transformation of (1): $v=u_{y}$. Substituting this transformation into (1), yields

$$
u_{y t}+a\left[2 u_{x} u_{y}+2 u u_{x y}+u_{x x y}\right]=0 .
$$

Now we apply the mapping approach to (6). By the balancing procedure, ansatz (3) becomes

$$
u(x, y, t)=f(x, y, t)+g(x, y, t) \phi(q),
$$

where $f, g$, and $q$ are functions of $(x, y, t)$ to be determined. Substituting (7) and (4) into (6) and collecting coefficients of the polynomials in $\phi$, then setting each coefficient to zero, we have

$$
f=-\frac{1}{2} \frac{q_{t}+a q_{x x}+a q_{x}^{2} \sigma}{a q_{x}}, \quad g=-q_{x},
$$

with

$$
q=\chi(x, t)+\varphi(y),
$$

where $\chi(x, t)$ and $\varphi(y)$ are two arbitrary functions of the indicated arguments.

Case 1. For $\sigma \neq 0$, we can derive the following solitary wave solutions and periodic wave solutions of (1):

$$
\begin{gathered}
u_{1}=-\frac{1}{2} \frac{\chi_{t}+a \chi_{x x}-a \chi_{x}^{2} \sigma \tanh \left[\frac{1}{2} \sigma(\chi+\varphi)\right]}{a \chi_{x}}, \\
v_{1}=-\frac{1}{4} \chi_{x} \varphi_{y} \sigma^{2}\left\{-1+\tanh \left[\frac{1}{2} \sigma(\chi+\varphi)\right]^{2}\right\} \\
u_{2}=-\frac{1}{2} \frac{\chi_{t}+a \chi_{x x}-a \chi_{x}^{2} \sigma \operatorname{coth}\left[\frac{1}{2} \sigma(\chi+\varphi)\right]}{a \chi_{x}}, \\
v_{2}=-\frac{1}{4} \chi_{x} \varphi_{y} \sigma^{2}\left\{-1+\operatorname{coth}\left[\frac{1}{2} \sigma(\chi+\varphi)\right]^{2}\right\} \\
u_{3}=-\frac{1}{2} \frac{\chi_{t}+a \chi_{x x}+a \chi_{x}^{2} \sigma \tan \left[\frac{1}{2} \sigma(\chi+\varphi)\right]}{a \chi_{x}}, \\
v_{3}=-\frac{1}{4} \chi_{x} \varphi_{y} \sigma^{2}\left\{1+\tan \left[\frac{1}{2} \sigma(\chi+\varphi)\right]^{2}\right\} \\
u_{4}=-\frac{1}{2} \frac{\chi_{t}+a \chi_{x x}-a \chi_{x}^{2} \sigma \cot \left[\frac{1}{2} \sigma(\chi+\varphi)\right]}{a \chi_{x}}, \\
v_{4}=-\frac{1}{4} \chi_{x} \varphi_{y} \sigma^{2}\left\{1+\cot \left[\frac{1}{2} \sigma(\chi+\varphi)\right]^{2}\right\}
\end{gathered}
$$

with two arbitrary functions being $\chi(x, t)$ and $\varphi(y)$.

Case 2. For $\sigma=0$, we can derive the following variable separated solution of (1):

$$
u_{5}=-\frac{1}{2} \frac{\chi_{t}+a \chi_{x x}}{a \chi_{x}}+\frac{\chi_{x}}{\chi+\varphi},
$$




$$
v_{5}=-\frac{\chi_{x} \varphi_{y}}{(\chi+\varphi)^{2}}
$$

with two arbitrary functions being $\chi(x, t)$ and $\varphi(y)$.

\section{Some Novel Folded Localized Excitations in the (2+1)-Dimensional VCBK System}

Due to the arbitrariness of the functions $\chi(x, t)$ and $\varphi(y)$ included in the above cases, the physical quantities $u$ and $v$ may possess rich structures. In this section, we mainly discuss some folded localized coherent excitations in the $(2+1)$-dimensional VCBK system. For simplicity, we only discuss the field $v_{2}$ of (13), namely

$$
\begin{aligned}
& V=v_{2}= \\
& -\frac{1}{4} \chi_{x} \varphi_{y} \sigma^{2}\left\{-1+\operatorname{coth}\left[\frac{1}{2} \sigma(\chi+\varphi)\right]^{2}\right\} .
\end{aligned}
$$

In order to construct kinds of interesting folded localized excitations, we introduce some suitable multivalued functions [26]. For instance,

$$
\chi_{x}=\sum_{j=1}^{M} V_{j}\left(\varepsilon-c_{j} t\right), \quad x=\varepsilon+\sum_{j=1}^{M} P_{j}\left(\varepsilon-c_{j} t\right)
$$

where $c_{j}(j=1,2, \cdots, M)$ are arbitrary constants, $V_{j}$ and $P_{j}$ are localized excitations with the properties $V_{j}( \pm \infty)=0, P_{j}( \pm \infty)=$ consts. We also treat the function $\varphi(y)$ in this way,

$$
\varphi_{y}=\sum_{j=1}^{N} U_{j}(\zeta), \quad y=\zeta+\sum_{j=1}^{N} Q_{j}(\zeta)
$$

Now we focus our attention on the intriguing evolution of two folded solitary waves for the solution $V$. If we select $\chi$ and $\varphi$ to be some appropriate multi-valued functions, then we can see that the interactions among the folded solitary waves are completely elastic. For instance, if we choose $\chi$ and $\varphi$ as

$$
\begin{aligned}
& \chi_{x}=-\operatorname{sech}(\varepsilon-t)^{2}-\operatorname{sech}(\varepsilon+t)^{2}, \\
& x=\varepsilon-0.6 \tanh (\varepsilon-t)-0.6 \operatorname{sech}(\varepsilon+t), \\
& \varphi_{y}=\operatorname{sech}(\zeta)^{2}, \quad y=\zeta-0.6 \tanh (\zeta),
\end{aligned}
$$

we can derive the time evolution of folded solitary waves for the physical quantity $V$ presented in Figure 1 with fixed parameters $\sigma=1$, at different times (a) $t=-3$, (b) $t=0$, (c) $t=3$.
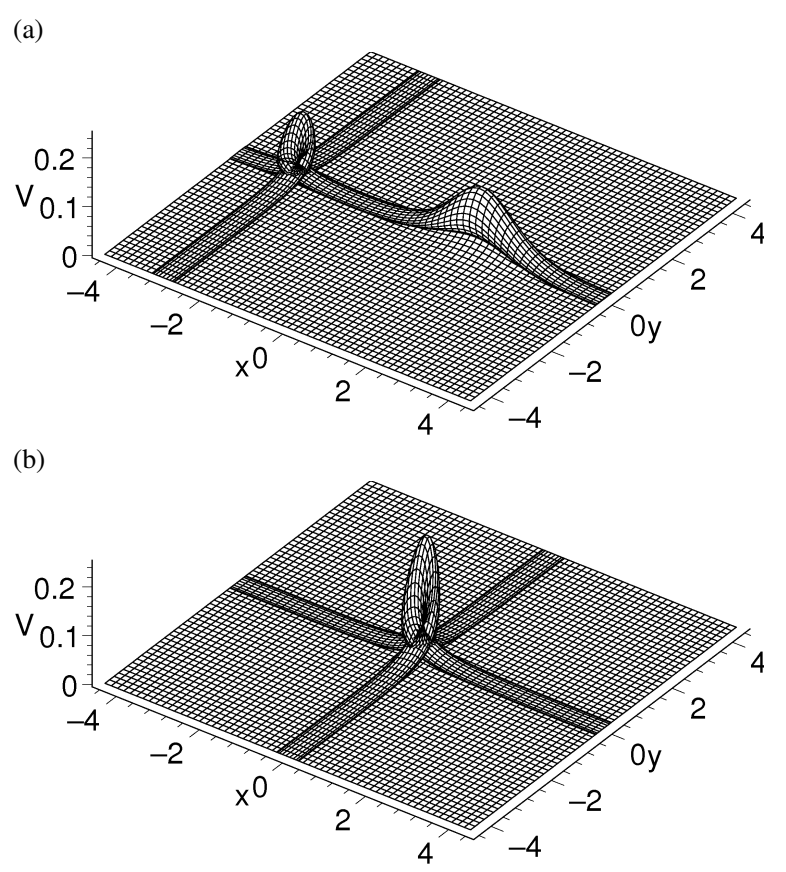

(c)

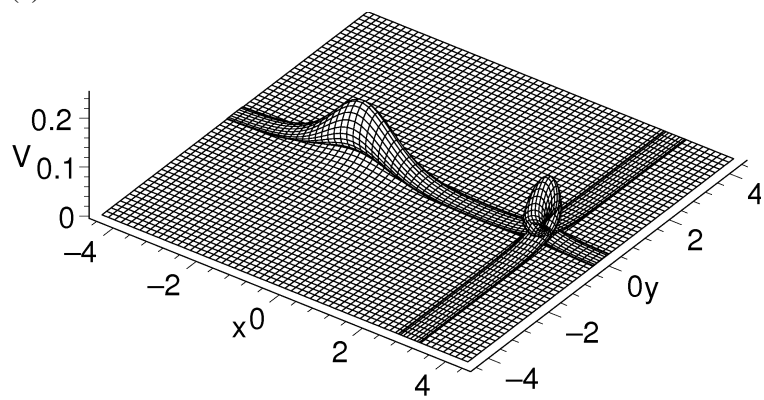

Fig. 1. Evolutional profile of the two folded solitary waves for the solution $V$ expressed by (20) with condition (23) at different times (a) $t=-3$, (b) $t=0$, (c) $t=3$, respectively.

If we choose $\chi$ and $\varphi$ as

$$
\begin{aligned}
& \chi_{x}=-\tanh (\varepsilon-t)^{2}-\operatorname{sech}(\varepsilon+t)^{2}, \\
& x=\varepsilon-0.8 \tanh (\varepsilon-t)-0.8 \operatorname{sech}(\varepsilon+t), \\
& \varphi_{y}=\operatorname{sech}(\zeta)^{2}, \quad y=\zeta-0.8 \operatorname{sech}(\zeta),
\end{aligned}
$$

we can derive another time evolution of folded solitary waves for the physical quantity $V$ presented in Figure 2 with fixed parameters $\sigma=1$, at different times (a) $t=-5$, (b) $t=-2.5$, (c) $t=0$,, (d) $t=2.5$, (e) $t=5$. From Figure 1 and 2, one can find the interactions between the two folded solitary waves are completely elastic since their amplitudes, velocities, and wave shapes do not undergo any change after their collision. 
(a)

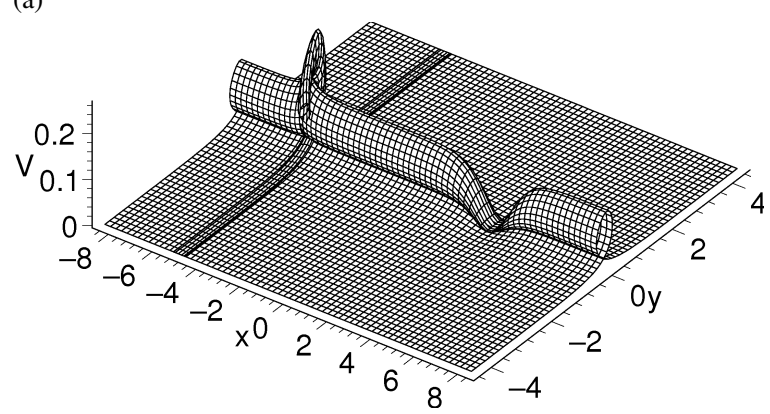

(c)

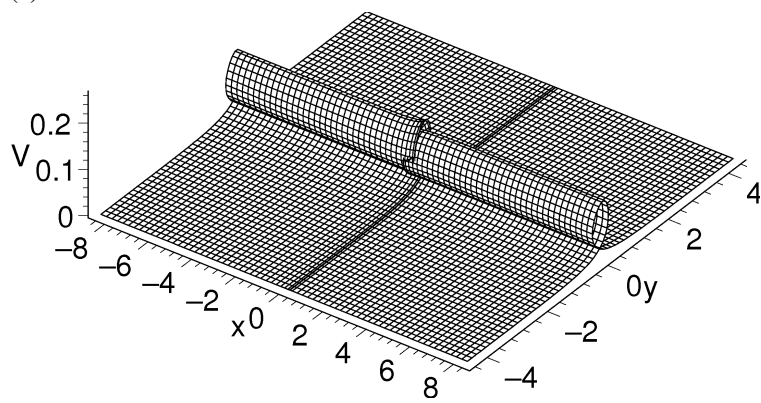

(e)

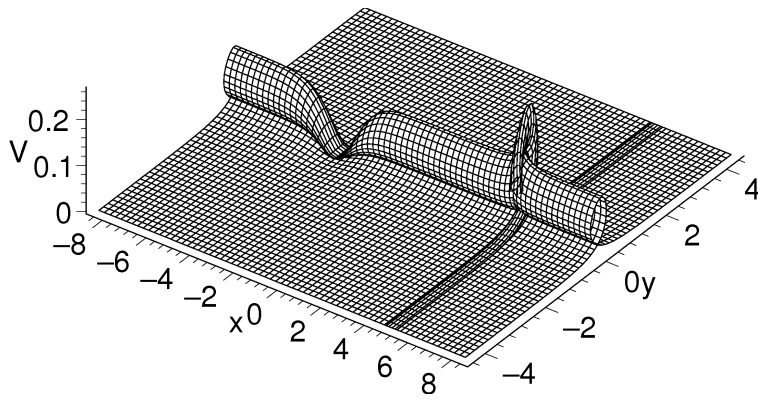

Furthermore, if we choose $\chi$ and $\varphi$ as

$$
\begin{aligned}
& \chi_{x}=-\operatorname{sech}(\varepsilon-t)^{2}-\operatorname{sech}(\varepsilon+t)^{2}, \\
& x=\varepsilon-0.2 \operatorname{sech}(\varepsilon-t)-0.2 \operatorname{sech}(\varepsilon+t), \\
& \varphi_{y}=\operatorname{sech}(\zeta)^{2}, \quad y=\zeta-0.2 \operatorname{sech}(\zeta),
\end{aligned}
$$

we can derive another time evolution of folded solitary waves for the physical quantity $V$ presented in Figure 3 with fixed parameters $\sigma=1$, at different times (a) $t=$ -3, (b) $t=-1.6$, (c) $t=-0.8$, (d) $t=-0.4$, (e) $t=0.3$, (f) $t=0.6$, (g) $t=1.6$, (h) $t=3$. From Figure 3 we can see that a rotation behaviour happens when the two folded solitary waves collide with each other, but their amplitudes, velocities, and wave shapes do not change after the collision.

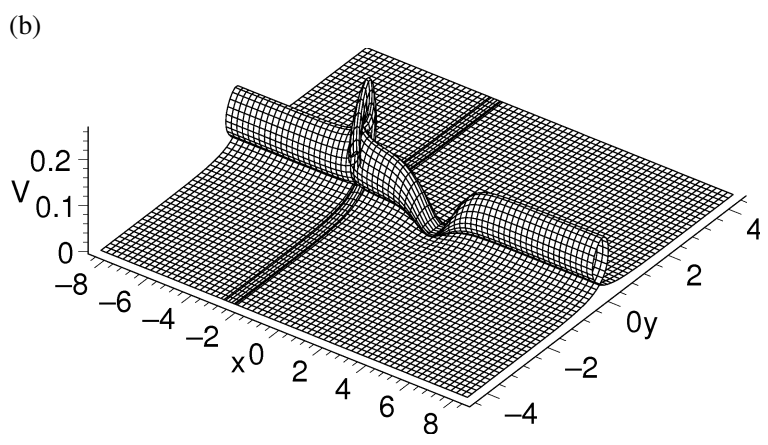

(d)

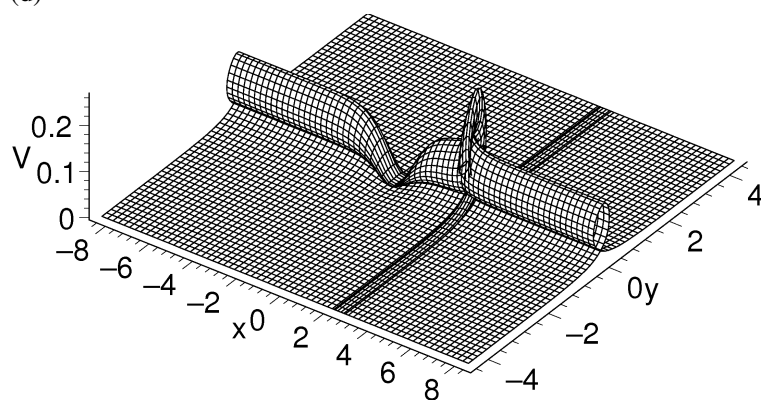

Fig. 2. Evolutional profile of the two folded solitary waves for the solution $V$ expressed by (20) with condition (24) at different times (a) $t=-5$, (b) $t=-2.5$, (c) $t=0$, (d) $t=2.5$, (e) $t=5$, respectively.

\section{Summary and Discussion}

In the past, many authors have found the exact solutions of some nonlinear systems via the Ricatti equation $\left(\phi^{\prime}=\sigma+\phi^{2}\right)$ mapping method. In summary, via a new projective equation $\left(\phi^{\prime}=\sigma \phi+\phi^{2}\right)$ and a linear variable separation approach, we find some new exact solutions of the $(2+1)$-dimensional Broek-Kaup system with variable coefficients, and based on the derived solitary wave solution $v_{2}$ of (13), we find a few novel folded solitary wave excitations. To our knowledge, the time evolutions of the two folded solitary waves displayed in Figure 2 and 3 are different from the ones presented in the previous work.

As to the application of this equation $\left(\phi^{\prime}=\sigma \phi+\phi^{2}\right)$ mapping approach to some other nonlinear systems, 
(a)

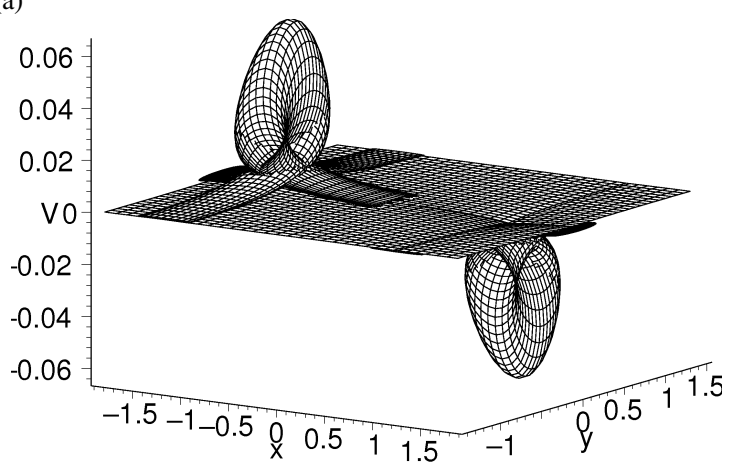

(c)

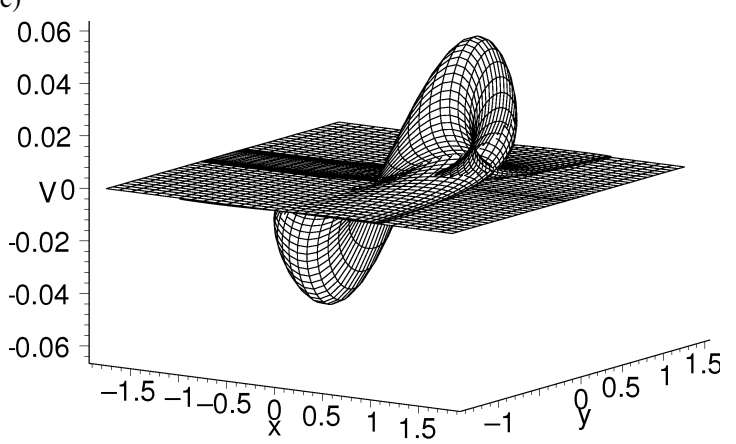

(e)

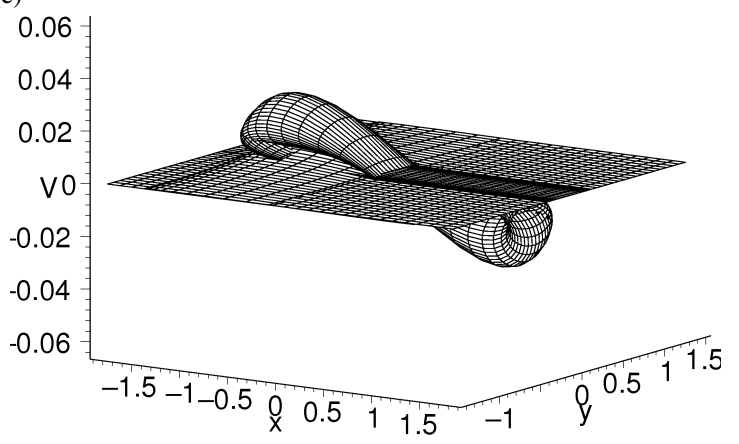

$(\mathrm{g})$

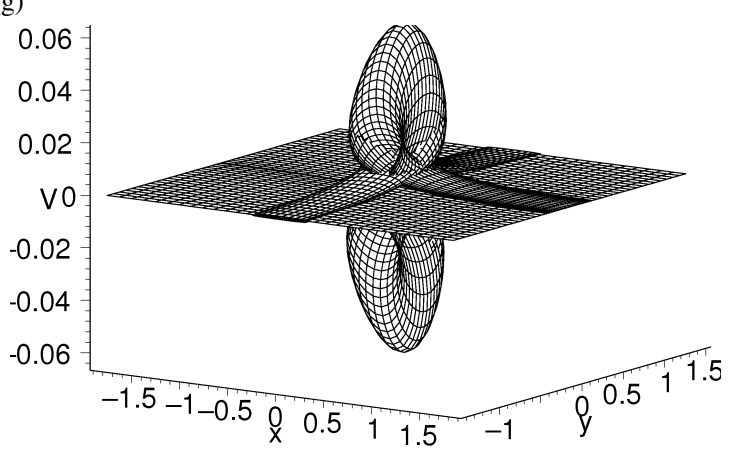

(b)

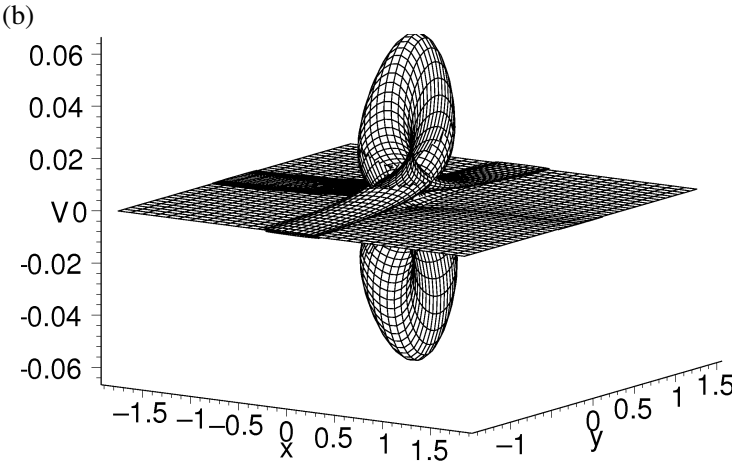

(d)

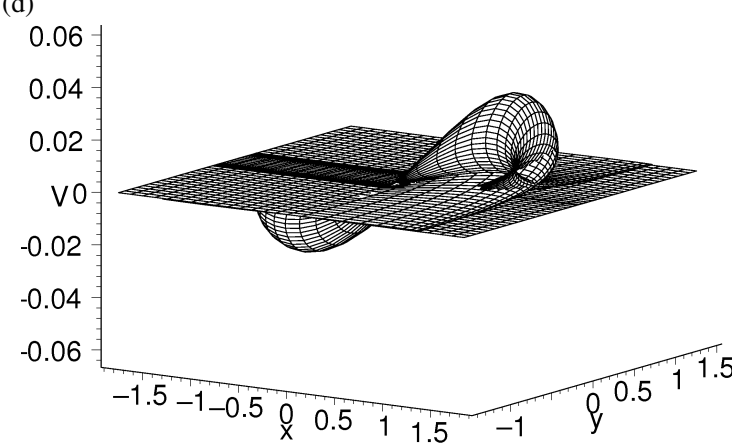

(f)

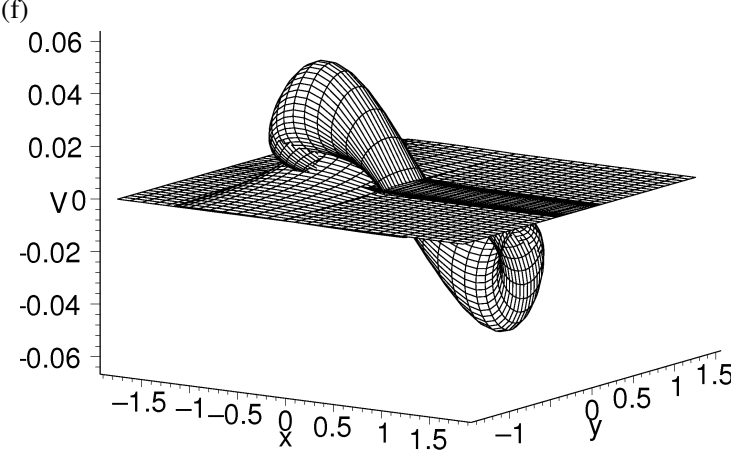

(h)

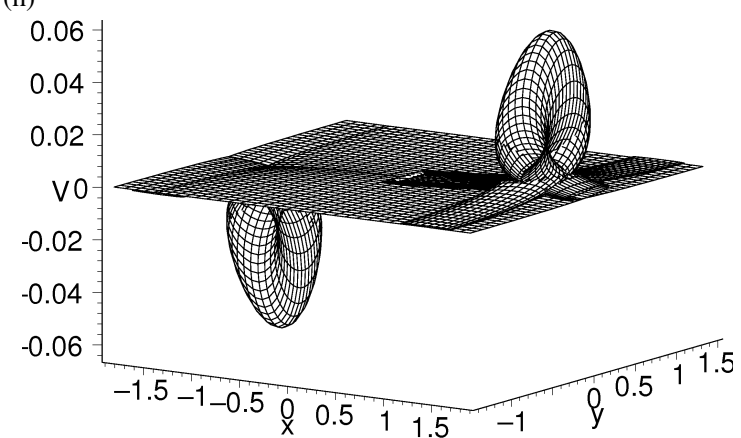

Fig. 3. A novel time evolution of folded solitary waves for the solution $V$ expressed by (20) with condition (25) at different times (a) $t=-3$, (b) $t=-1.6$, (c) $t=-0.8$, (d) $t=-0.4$, (e) $t=0.3$, (f) $t=0.6$, (g) $t=1.6$, (h) $t=3$, respectively. 
we will carry out a further investigation in our future work.

\section{Acknowledgements}

The authors would like to thank Professor JieFang Zhang for his fruitful and helpful suggestions. This work has been supported by the Natural Science Foundation of Zhejiang Province (Grant

[1] S. Y. Lou and X. Y. Tang, Chin. Phys. Lett. 19, 769 (2002).

[2] I. Loutsenko and D. Roubtsov, Phys. Rev. Lett. 78, 3001 (1997).

[3] M. Tajiri and H. Maesono, Phys. Rev. E 55, 3351 (1997).

[4] D. W. C. Lai and K. W. Chow, J. Phys. Soc. Jpn. 70, 666 (2001).

[5] V. N. Serkin, Opt. Commun. 192, 237 (2001).

[6] V.E. Zakharov and J. Li, Appl. Mech. Tech. Phys. 9, 190 (1998).

[7] S. Wang, X. Y. Tang, and S. Y. Lou, Chaos, Solitons, and Fractals 19, 769 (2004).

[8] S. Y. Lou, Commun. Theor. Phys. (Beijing, China) 26, 487 (1996).

[9] S. Y. Lou, J. Lin, and X. Y. Tang, Eur. Phys. J. B22, 473 (2001).

[10] D. W. C. Lai and K. W. Chow, J. Phys. Soc. Jpn. 70, 666 (2001).

[11] J. F. Zhang, Commun. Theor. Phys. (Beijing, China) 37, 277 (2002).

[12] J.P. Fang and C.L. Zheng, Z. Naturforsch. 60a, 245 (2005).

[13] J. P. Fang and C. L. Zheng, Acta Phys. Sin. 54, 2991 (2005).
Nos. Y604106, Y606128), the Scientific Research Fund of Zhejiang Provincial Education Department of China (Grant No. 20070568), the undergraduate innovation project of science and technology of Zhejiang Province, the project of seeding grants of Zhejiang Province, and Natural Science Foundation of Zhejiang Lishui University (Grant No. KZ09005).

[14] J. P. Fang and C. L. Zheng, Z. Naturforsch. 60a, 250 (2005).

[15] S.H. Ma, X.H. Wu, J.P. Fang, and C.L. Zheng, Z. Naturforsch. 61a, 249 (2006).

[16] S. H. Ma and J. P. Fang, Acta. Phys. Sin. 55, 37 (2006).

[17] S. H. Ma, J. P. Fang, and C. L. Zheng, Z. Naturforsch. 62a, 8 (2007).

[18] S. H. Ma, J. Y. Qiang, and J. P. Fang, Acta. Phys. Sin. 56, 0620 (2007).

[19] S. H. Ma, J. P. Fang, and H. P. Zhu, Acta. Phys. Sin. 56, 4319 (2007).

[20] S. H. Ma, J. Y. Qiang, and J. P. Fang, Commun. Theor. Phys. (Beijing, China) 48, 662 (2007).

[21] S. H. Ma, J.P. Fang, and C. L. Zheng, Z. Naturforsch. 63a, 121 (2008).

[22] J. B. Li, C. L. Zheng, and S. H. Ma, Z. Naturforsch. 63a, 641 (2008).

[23] R. Conte and M. Musette, J. Phys. A Math. Gen. 25, 5609 (1992).

[24] Z. T. Fu, S. D. Liu, and S. K. Liu, Chaos, Solitons, and Fractals 20, 301 (2004).

[25] J. P. Ying, S. Y. Lou, Z. Naturforsh. 56a, 619 (2001).

[26] X. Y. Tang and S. Y. Lou, Commun. Theor. Phys. 40, $62(2003)$. 